\title{
Demanda em Fonoaudiologia em um serviço público municipal da região Sul do Brasil
}

\author{
Demand in Speech-Language Pathology and Audiology in a \\ public service in the South region of Brazil
}

\author{
Roseris Denicol Diniz ${ }^{1}$, Ronaldo Bordin ${ }^{2}$
}

\begin{abstract}
RESUMO
Objetivo: Caracterizar a demanda encaminhada a um Serviço de Fonoaudiologia de Centro de Saúde, no biênio 2006/2007. Métodos: Estudo de demanda, transversal, utilizando-se o banco de dados do referido serviço e o registro de prontuários. A partir da coleta de dados no banco de dados do serviço obtiveram-se as seguintes variáveis: gênero, idade e origem do encaminhamento. Nos prontuários foram codificados os motivos da consulta (queixa inicial) e a hipótese diagnóstica. Resultados: Do total de atendimentos registrados ( $\mathrm{n}=243)$, houve predomínio do gênero masculino $(65 \%)$, da faixa etária de seis aos 12 anos $(52,3 \%)$, com encaminhamento por médico pediatra $(35,8 \%)$, queixa de alteração de fala $(67,8 \%)$ e hipótese diagnóstica de desvio fonético (28,4\%); 37 prontuários (15,2\%) não apresentavam indicação para tratamento e 31 (12,8\%) não registravam hipótese diagnóstica. Conclusão: É necessário um maior investimento no planejamento de ações em saúde fonoaudiológica, visando que as alterações de fala diminuam a médio e longo prazo, assim como as listas de espera para tal especialidade, e que os registros em prontuários e encaminhamentos para esta área ocorram de forma mais qualificada.
\end{abstract}

Descritores: Ciências da saúde; Saúde pública; Gestão em saúde; Epidemiologia; Necessidades e demandas de serviços de saúde

\section{INTRODUÇÃO}

É fundamental considerar-se os aspectos da fala, da linguagem e da audição como atributos da saúde. Suas manifestações patológicas comprometem a competência e o desempenho comunicativo verbal, não verbal, intra e interpessoal, geram sofrimento e insucesso social e, interferem na qualidade de vida dos indivíduos ${ }^{(1)}$. Por meio da comunicação o indivíduo atua como agente transformador da sociedade e da sua realidade e por isto, a comunicação merece atenção na saúde pública ${ }^{(2,3)}$.

A Fonoaudiologia vem ampliando seus campos de inserção. Em relação à saúde pública, porém, ainda necessita de estudos que produzam conhecimentos técnicos e científicos que expandam seu domínio de atuação(4).

A introdução do fonoaudiólogo no sistema de saúde pública foi marcada por contratempos que conduziram os profissionais à opção pela intervenção clínica. Tal opção resultou no

Trabalho realizado na Faculdade de Medicina, Departamento de Medicina Social, Universidade Federal do Rio Grande do Sul - UFRGS - Porto Alegre (RS), Brasil.

(1) Prefeitura Municipal de Porto Alegre - Porto Alegre (RS), Brasil.

(2) Departamento de Medicina Social da Faculdade de Medicina, Universidade Federal do Rio Grande do Sul - UFRGS - Porto Alegre (RS), Brasil.

Endereço para correspondência: Roseris Denicol Diniz. R. Antonio Ricardo

Silva Vargas, 110, Dom Feliciano, Gravataí (RS), Brasil, CEP: 94015-141.

E-mail: roseris.diniz@ig.com.br

Recebido em: 12/8/2009; Aceito em: 14/10/2010 isolamento deste profissional ${ }^{(5)}$. Na década de 90 houve uma ampliação na atuação fonoaudiológica para além da clínica terapêutica, enfatizando as alterações da linguagem com ações dirigidas diretamente para a população ${ }^{(2)}$.

O fonoaudiólogo na saúde coletiva deve ser comprometido com os princípios de humanização, acolhimento, vínculo e responsabilidade pelo território em que atua e pela comunidade que nele vive ${ }^{(6)}$. Este já é um profissional conhecido, importante e integrante das equipes que atuam em saúde coletiva( ${ }^{(7)}$.

A atuação da Fonoaudiologia na saúde coletiva requer a formação de profissionais com visão de promoção da saúde, no sentido de que podem contribuir para a melhoria da qualidade de vida dos cidadãos, uma vez que a saúde e a qualidade de vida estão no foco da saúde fonoaudiológica ${ }^{(8,9)}$.

É preciso conhecer as condições de saúde da coletivida$\mathrm{de}^{(2)}$, por meio do diagnóstico das necessidades em saúde, a fim de definir ações para os problemas de maior ocorrência na população ${ }^{(10)}$ uma vez que a demanda, na sociedade atual, é correntemente maior do que a disponibilidade de recursos humanos, tecnológicos e financeiros, entre outros ${ }^{(11)}$.

É importante, também, que estudos epidemiológicos relacionados aos distúrbios da comunicação sejam realizados e publicados, explicitando principalmente termos técnicos e formas de busca de tais informações ${ }^{(12)}$. Assim como são importantes a utilização de nomenclatura mais homogênea e a criação de indicadores padronizados de avaliação e moni- 
toramento das ações fonoaudiológicas, a fim de melhorar a precisão destas ações e propiciar a comparação de estudos de diferentes regiões ${ }^{(11,13)}$.

Diante disto, este estudo tem por objetivo caracterizar a demanda encaminhada para o serviço de Fonoaudiologia de um Centro de Saúde da rede municipal de atenção à saúde do município de Porto Alegre (RS), no biênio 2006-2007.

\section{MÉTODOS}

No município de Porto Alegre, capital do estado do Rio Grande do Sul (RS), o cargo de Fonoaudiólogo foi criado em 1996, com as 11 vagas totalmente preenchidas apenas em 2000, sendo que nove profissionais atuam na área de fonoterapia $\mathrm{e}$ dois na área de audiologia.

Porto Alegre possui 16 Distritos Sanitários agrupados em oito Gerências Distritais. Os Serviços de Fonoaudiologia do município estão centralizados em quatro Centros de Saúde, ligados à área das especialidades, cada um responsável pelo atendimento da população de determinadas Gerências Distritais, distribuídas deste modo em função da proximidade dos Distritos Sanitários e na tentativa de não deixar áreas descobertas na saúde fonoaudiológica. Cada um dos Serviços de Fonoaudiologia possui sistemáticas diferentes de atendimento e funcionamento, como por exemplo, o acesso ao Serviço e as modalidades de atendimento. No final de 2009 encontravam-se em lista de espera para esta especialidade em torno de 1033 pessoas $^{(14)}$.

O Serviço de Fonoaudiologia do Centro de Saúde estudado conta com três fonoaudiólogas na área de fonoterapia e duas na de audiologia. O Serviço de Audiologia abrange todo o município, enquanto que o de Fonoterapia atende duas Gerências Distritais (GD) Centro e Lomba do Pinheiro/Partenon, correspondendo a uma população de 266.896 e 176.613 habitantes, respectivamente, totalizando 443.504 habitantes de uma população total de 1.360 .739 habitantes (IBGE, 2000) ${ }^{(15)}$.

Os encaminhamentos para o Serviço de Fonoaudiologia deste Centro de Saúde na área de fonoterapia são realizados por qualquer profissional da rede de saúde através de ficha de referência e contra-referência, sem restrição de patologias.

O estudo foi realizado a partir dos dados da totalidade de usuários atendidos no Serviço de Fonoaudiologia - área de Fonoterapia do Centro de Saúde Santa Marta do município de Porto Alegre - RS, no biênio 2006-2007 ( $\mathrm{n}=243$ casos registrados), utilizando-se o banco de dados existente no serviço e os registros em prontuários. Assim, se constitui em um estudo de demanda, transversal, descrevendo os atendimentos registrados em um serviço de saúde específico.

Ainda que os dados estivessem à disposição, a análise dos mesmos, focando na administração e planejamento das ações em saúde fonoaudiológica, não é costumeiramente realizada nos serviços municipais de saúde.

As seguintes variáveis foram obtidas no banco de dados: idade dos usuários quando do ingresso no serviço, gênero e origem do encaminhamento. No que se refere à origem do encaminhamento (quem encaminhou), foi empregado o seguinte critério: pediatra, clínico geral, otorrinolaringologista, neurologista, neuropediatra, profissional da área de saúde mental (psicólogo, psiquiatra, psicopedagogo, terapeuta familiar, fonoaudiólogo) ou do programa de saúde do escolar, entre outros.

Nos prontuários, foram codificados os motivos da consulta fonoaudiológica (queixa inicial) apresentados pelo responsável e ou usuário na primeira consulta, assim como a hipótese diagnóstica firmada. Foram consideradas alterações na área da motricidade oral as anatômicas e/ou funcionais do sistema sensório motor oral, que comprometem as funções realizadas pelo sistema estomatognático de mastigação, sucção, deglutição, respiração e fala ${ }^{(16)}$. Incluem-se nesta as fissuras, disfagias, gagueira, respiração oral, entre outras patologias fonoaudiológicas.

Destaca-se que desvio fonético refere-se às alterações que se restringem ao domínio do padrão fonêmico da língua, correspondendo à realização motora da linguagem; compreende as alterações que afetam a produção dos sons da língua, ligados às fases de programação e execução neuromotora. Desvio fonológico, por seu lado, corresponde a dificuldades que dizem respeito ao domínio do padrão fonêmico da língua, na ausência de alterações orgânicas detectáveis como deficiência auditiva e anormalidades anatômicas ou neurofisiológicas ${ }^{(17)}$.

As alterações na área da linguagem dizem respeito àquelas que comprometem o uso da linguagem para fins comunicativos, a capacidade de produzir e compreender enunciados gramaticalmente estruturados e ou semanticamente apropriados, sendo que, na criança, afetam todo o seu desenvolvimento linguístico $^{(17)}$. Encontra-se nesta categoria o atraso de linguagem, a afasia e o distúrbio de aprendizagem. Compreendem alteração na área da voz (disfonias), as dificuldades na emissão da voz com suas características normais ${ }^{(18)}$.

A análise dos dados empregou estatística descritiva (frequência e percentuais), média e mediana para a idade, com medidas de associação realizadas pelos testes do qui-quadrado e t de Student, para uma significância de 5\% e poder de estudo de $80 \%$.

Este estudo foi aprovado pelo Comitê de Ética e Pesquisa da Secretaria Municipal de Saúde de Porto Alegre sob Registro $n^{\circ}$. 317, Processo n ${ }^{\circ}$. 001.065015.08.8, e da instituição de ensino superior ao qual está vinculado, protocolo $\mathrm{n}^{\circ} .14739$.

\section{RESULTADOS}

Dos 243 prontuários analisados, $158(65 \%)$ envolviam indivíduos do gênero masculino e 85 (35\%) do feminino. A idade média de idade variou de 1 a 86 anos, com mediana de 7 e média de 11,96 anos. Por gênero, a idade média entre os homens foi de $10,01 \pm 11,75$; sendo superior entre as mulheres, de $15,58 \pm 20,46(\mathrm{p}=0,023)$.

Na Tabela 1 observa-se que o predomínio dos encaminhamentos deu-se em menores de 13 anos de idade ( $84 \%$ do total), sendo 52,3\% correspondentes à faixa etária dos 6 aos 12 anos de idade e $28,8 \%$ dos 3 aos 5 anos de idade. O encaminhamento de pacientes com idade superior a 60 anos foi de $4,1 \%$.

Na Tabela 2 encontra-se relacionado o profissional responsável pelo encaminhamento. A maior parte destes foi realizada por médico pediatra $(35,8 \%)$ seguida de profissional vinculado ao Programa Saúde do Escolar $(15,2 \%)$ e por otorrinolarin- 
Tabela 1. Distribuição por faixa etária dos pacientes encaminhados ao Serviço de Fonoaudiologia - área de fonoterapia do Centro de Saúde Santa Marta, Porto Alegre (RS), em 2006-2007

\begin{tabular}{lcc}
\hline Faixa etária (anos) & $\mathrm{n}$ & $\%$ \\
\hline $0-2$ & 7 & 2,9 \\
$3-5$ & 70 & 28,8 \\
$6-12$ & 127 & 52,3 \\
$13-18$ & 7 & 2,9 \\
$19-60$ & 22 & 9,1 \\
$\geq 61$ & 10 & 4,1 \\
\hline Total & 243 & 100 \\
\hline
\end{tabular}

Legenda: $\mathrm{n}=$ casos registrados

gologista (14,8\%). Não ocorreu diferença significativa entre a origem e o gênero do usuário encaminhado $(\mathrm{p}=0,45$ para as cinco principais origens).

Os motivos principais da consulta com a área da Fonoaudiologia, ou seja, a queixa apresentada na primeira consulta pelo paciente e ou responsável, estão registrados (um por caso) na Tabela 3. Do total de prontuários analisados, os motivos de consulta mais frequentes foram alterações de fala $(67,5 \%)$ e atraso de linguagem $(7 \%)$.

Não ocorreu diferença significativa entre o motivo da consulta e gênero (Tabela 3) $(\mathrm{p}=0,18)$. Em se considerando exclusivamente os dois principais motivos de consulta, ocorreu diferença significativa por gênero $(\mathrm{p}=0,02)$ e borderline por faixa etária $(\mathrm{p}=0,06)$. Dos 164 motivos envolvendo alteração de fala, 143 ocorreram em menores de 13 anos de idade; das 17 queixas de atraso de linguagem, 16 também ocorreram nesta faixa etária. Enquanto os motivos da consulta por alteração de fala se concentraram na faixa entre 6 e 12 anos de idade, os de atraso de linguagem foram mais precoces, em menores de 6 anos de idade (OR=0,38 - 0,11 - 1,02).

A principal hipótese diagnóstica fonoaudiológica registrada encontra-se na Tabela 4. Do total de 243 prontuários, 31 $(12,8 \%)$ não apresentavam hipótese registrada e $37(15,2 \%)$ não apresentavam indicação para tratamento fonoaudiológico. Não ocorreu diferença significativa entre a hipótese firmada e o gênero, enquanto para a idade, os menores de 13 anos receberam $178(83,9 \%)$ das 212 hipóteses firmadas - 60/69 de desvio fonético, 51/54 de desvios fonético e fonoaudiológico e 26/27 dos atrasos de linguagem, com maior concentração destes entre as crianças entre 6 e 12 anos de idade $(\mathrm{p}=0,043)$.

Entre os 37 encaminhamentos sem indicação para tratamento fonoaudiológico, 24 (64,9\%) ocorreram no gênero masculino, idade média total de 17,8 anos e mediana de 10 anos de idade (20 casos na faixa etária entre 6 e 12 anos), sendo que $9(24,3 \%)$ foram realizados por pediatra e $6(16,2 \%)$ por profissional do programa de saúde do escolar. A relação entre encaminhamentos realizados e inexistência de indicação, para os três principais locais de procedência, foi de $10,3 \%$ para os

Tabela 2. Distribuição por origem de encaminhamento e gênero dos pacientes encaminhados ao Serviço de Fonoaudiologia - área de fonoterapia do Centro de Saúde Santa Marta, Porto Alegre (RS), em 2006-2007

\begin{tabular}{|c|c|c|c|c|c|c|}
\hline \multirow[t]{2}{*}{ Origem do encaminhamento } & \multicolumn{2}{|c|}{ Gênero masculino } & \multicolumn{2}{|c|}{ Gênero feminino } & \multicolumn{2}{|c|}{ Total } \\
\hline & $\mathrm{n}$ & $\%$ & $\mathrm{n}$ & $\%$ & $\mathrm{n}$ & $\%$ \\
\hline Pediatra & 52 & 32,9 & 35 & 41,2 & 87 & 35,8 \\
\hline Profissional do Programa de Saúde do Escolar & 27 & 17,1 & 10 & 11,8 & 37 & 15,2 \\
\hline Otorrinolaringologista & 27 & 17,1 & 9 & 10,6 & 36 & 14,8 \\
\hline Clínico geral & 20 & 12,7 & 10 & 11,8 & 30 & 12,3 \\
\hline $\begin{array}{l}\text { Encaminhamento interno da equipe de saúde mental do Centro } \\
\text { de Saúde Santa Marta }\left(^{*}\right)\end{array}$ & 12 & 7,6 & 7 & 8,2 & 19 & 7,8 \\
\hline Odontólogo & 1 & 0,6 & 4 & 4,7 & 5 & 2,1 \\
\hline Neurologista & 2 & 1,3 & 3 & 3,5 & 5 & 2,1 \\
\hline Neuropediatra & 3 & 1,9 & 1 & 1,2 & 4 & 1,6 \\
\hline Psicólogo & 3 & 1,9 & 0 & 0,0 & 3 & 1,2 \\
\hline Médico de família & 1 & 0,6 & 2 & 2,4 & 3 & 1,2 \\
\hline Sem ficha de referência e contra-referência & 1 & 0,6 & 2 & 2,4 & 3 & 1,2 \\
\hline Assistente social & 2 & 1,3 & 0 & 0,0 & 2 & 0,8 \\
\hline Pneumologista & 2 & 1,3 & 0 & 0,0 & 2 & 0,8 \\
\hline Psiquiatra & 2 & 1,3 & 0 & 0,0 & 2 & 0,8 \\
\hline Terapeuta ocupacional & 1 & 0,6 & 1 & 1,2 & 2 & 0,8 \\
\hline Conselheiro tutelar & 1 & 0,6 & 0 & 0,0 & 1 & 0,5 \\
\hline Promotor de justiça & 1 & 0,6 & 0 & 0,0 & 1 & 0,5 \\
\hline Sem identificação & 0 & 0,0 & 1 & 1,2 & 1 & 0,5 \\
\hline Total & 158 & 100 & 85 & 100 & 243 & 100 \\
\hline
\end{tabular}

$\left(^{*}\right)$ Profissionais da equipe de Saúde Mental do Centro de Saúde Santa Marta: psicólogo, psicopedagogo, psiquiatra e terapeuta de família Legenda: $\mathrm{n}=$ casos registrados 
Tabela 3. Distribuição por motivo da consulta e gênero dos pacientes encaminhados ao Serviço de Fonoaudiologia - área de fonoterapia do Centro de Saúde Santa Marta, Porto Alegre (RS), em 2006-2007

\begin{tabular}{|c|c|c|c|c|c|c|}
\hline \multirow{2}{*}{ Motivo da consulta } & \multicolumn{2}{|c|}{ Gênero masculino } & \multicolumn{2}{|c|}{ Gênero feminino } & \multicolumn{2}{|c|}{ Total } \\
\hline & $\mathrm{n}$ & $\%$ & $\mathrm{n}$ & $\%$ & $\mathrm{n}$ & $\%$ \\
\hline Alteração de fala & 113 & 71,5 & 51 & 60 & 164 & 67,5 \\
\hline Atraso de linguagem & 12 & 7,6 & 5 & 5,9 & 17 & 7 \\
\hline Alteração de motricidade oral & 7 & 4,4 & 5 & 5,9 & 12 & 4,9 \\
\hline Problema de voz & 4 & 2,6 & 8 & 9,4 & 12 & 4,9 \\
\hline Encaminhado pela escola & 7 & 4,4 & 5 & 5,9 & 12 & 4,9 \\
\hline Gagueira & 7 & 4,4 & 5 & 5,9 & 12 & 4,9 \\
\hline Sem descrição no prontuário & 2 & 1,3 & 4 & 4,6 & 6 & 2,5 \\
\hline Encaminhado pelo médico & 2 & 1,3 & 2 & 2,4 & 4 & 1,6 \\
\hline Problema de audição & 2 & 1,3 & 0 & 0 & 2 & 0,8 \\
\hline Distúrbio de aprendizagem & 1 & 0,6 & 0 & 0 & 1 & 0,5 \\
\hline Não sabe & 1 & 0,6 & 0 & 0 & 1 & 0,5 \\
\hline Total & 158 & 100 & 85 & 100 & 243 & 100 \\
\hline
\end{tabular}

Legenda: $\mathrm{n}=$ casos registrados

Tabela 4. Distribuição por hipótese diagnóstica fonoaudiológica principal e gênero dos pacientes encaminhados ao Serviço de Fonoaudiologia - Área de Fonoterapia do Centro de Saúde Santa Marta, Porto Alegre (RS), em 2006-2007

\begin{tabular}{|c|c|c|c|c|c|c|}
\hline \multirow{2}{*}{ Hipótese diagnóstica } & \multicolumn{2}{|c|}{ Gênero masculino } & \multicolumn{2}{|c|}{ Gênero feminino } & \multicolumn{2}{|c|}{ Total } \\
\hline & $\mathrm{n}$ & $\%$ & $\mathrm{n}$ & $\%$ & $\mathrm{n}$ & $\%$ \\
\hline Desvio fonético & 45 & 28,5 & 24 & 28,2 & 69 & 28,4 \\
\hline Desvio fonético/fonológico & 36 & 22,8 & 18 & 21,2 & 54 & 22,2 \\
\hline Sem indicação & 24 & 15,2 & 13 & 15,3 & 37 & 15,2 \\
\hline Sem hipótese diagnóstica & 19 & 12,0 & 12 & 14,1 & 31 & 12,8 \\
\hline Atraso de linguagem & 17 & 10,8 & 10 & 11,8 & 27 & 11,1 \\
\hline Gagueira & 7 & 4,4 & 2 & 2,4 & 9 & 3,8 \\
\hline Alteração do sistema motor oral & 4 & 2,5 & 4 & 4,7 & 8 & 3,3 \\
\hline Disfagia & 4 & 2,5 & 0 & 0,0 & 4 & 1,6 \\
\hline Disfonia & 1 & 0,6 & 1 & 1,2 & 2 & 0,8 \\
\hline Fissura & 1 & 0,6 & 0 & 0,0 & 1 & 0,4 \\
\hline Afasia & 0 & 0,0 & 1 & 1,2 & 1 & 0,4 \\
\hline Total & 158 & 100 & 85 & 100 & 243 & 100 \\
\hline
\end{tabular}

Legenda: $\mathrm{n}=$ casos registrados

pediatras, 16,2\% para os profissionais do Programa Saúde do Escolar e 8,3\% para os otorrinolaringologistas.

\section{DISCUSSÃO}

Os prontuários analisados apresentam uma predominância do gênero masculino, fato que coincide com vários levantamentos da literatura nesta área ${ }^{(2,19-22)}$. Entretanto, sugerem que esta prevalência elevada no gênero masculino seja estudada, já que nenhuma discussão mais aprofundada foi ainda realizada ${ }^{(2)}$.

Outros estudos também indicam o predomínio de alteração na fala superior no gênero masculino ${ }^{(22,23)}$, destacando-se um destes, que apresenta a proporção de quatro homens para cada mulher com tal problema ${ }^{(24)}$. Apenas em um estudo foi encontrada uma proporção semelhante entre os gêneros, de aproximadamente $1: 1^{(13)}$. Uma potencial explicação seria que a aquisição e o desenvolvimento da linguagem ocorrem de forma diferente entre meninos e meninas, principalmente devido à forma distinta de interação entre o meio e a forma de criação, considerando o meio ambiente como um dos fatores associados aos agravos à saúde ${ }^{(12)}$.

Em relação à faixa etária, houve predomínio nas idades de 6 a 12 anos, seguido de 3 a 5 anos, ou seja, 81,1\% dos encaminhamentos ocorreu com crianças em idade escolar e préescolar. Muitos autores relacionam a ocorrência de problemas fonoaudiológicos em faixas etárias similares ${ }^{(20,23)}$ ou mesmo superiores $^{(19)}$. Acredita-se que os encaminhamentos nestas faixas etárias devam-se ao fato de ser a época que se iniciam as novas relações sociais da criança e os pais ou professores ficam mais atentos à questão da linguagem da criança. Ou seja, as crianças são mais cobradas quanto ao seu desempenho na linguagem oral e também na aprendizagem. Este alerta de pais e professores ocorre também em consequência da crença de que as crianças que não "falam bem" terão dificuldades na alfabetização ${ }^{(2)}$. Existe também uma crença muito difundida de que não se deve ficar preocupado se a criança não começa 
a falar na idade esperada, pois é normal que algumas falem mais tarde, sendo as mesmas encaminhadas aos profissionais com idades mais avançadas ${ }^{(20)}$.

Considera-se significativo o número de crianças encaminhadas com idade inferior a cinco anos neste Centro de Saúde, pois nenhum trabalho mais específico de orientação foi realizado nesta área. Em contrapartida, destaca-se a deficiência no encaminhamento para esta especialidade de adultos, principalmente de idosos, já que este serviço está ligado ao nível secundário de atenção à saúde - ainda que em patamares superiores aos relatados em outros estudos, de $12,6 \%$ e $6 \%{ }^{(2,19)}$.

Acredita-se que os encaminhamentos de adultos para atendimento nesta área são ainda baixos, pelo pouco conhecimento do fazer fonoaudiológico e suas áreas de atuação com esta população específica, tanto por parte dos profissionais como da população em geral, e também, pela crença popular da fonoaudiologia estar ligada à criança e a problemas de fala. O fato é corroborado pelo maior número de encaminhamentos realizados pelo pediatra, seguido do profissional do programa de saúde do escolar, otorrinolaringologista e clínico geral. $\mathrm{O}$ percentual de encaminhamentos realizados pelos profissionais de saúde foi semelhante aos encontrados em outro estudo, de $30,8 \%{ }^{(21)}$, diferindo dos que referiram maior ocorrência de encaminhamentos feitos por parte da escola, seguidos pelos feitos por postos de saúde ${ }^{(2,19)}$.

A escola funciona como um filtro, identificando os casos de dificuldades na comunicação que não foram detectados previamente e poderiam comprometer o desenvolvimento adequado das habilidades pré-linguísticas e o aprendizado da leitura e escrita.

O maior índice de encaminhamentos feito pela área médica deve-se, provavelmente, ao fato de esta ser uma das portas de entrada no sistema público de saúde, não sendo possível uma busca espontânea ao serviço ou encaminhamento direto da escola. Os dados apresentados em outros trabalhos foram realizados em locais distintos e com diferentes formas de acesso $^{(2,19,21,25)}$, ainda que em estudos mais recentes da área da saúde não ocorra busca espontânea neste tipo de atendimento.

As alterações de fala tiveram maior ocorrência como o principal motivo da consulta com a Fonoaudiologia, pela maior mobilização ou mesmo motivação por parte da família em busca de tratamento nas alterações da comunicação oral. Em outro estudo as alterações de fala também foram as queixas mais apresentadas (46\%), seguidas das de linguagem (18\%) ${ }^{(23)}$, coincidindo com os resultados do presente estudo, em que o atraso de linguagem foi a segunda queixa mais encontrada.

Entretanto, quanto à queixa de alteração na área de motricidade oral, os estudos divergem. Outros autores encontraram $15 \%{ }^{(23)}$ de queixas nesta área, ao passo que no estudo atual, a referência a alterações na área foi menor e coincidiu, em proporção, com outras queixas, como: problemas de voz, gagueira e encaminhamentos pela escola.

Na prática fonoaudiológica, a queixa de fala está em maior evidência dada sua grande importância para a plena efetividade de comunicação entre os seres humanos e, também, por existir uma expectativa dos pais em relação à aquisição e ao desenvolvimento da mesma. Em um estudo com 30 prontuários de pacientes que apresentavam alguma queixa de fala, as autoras encontraram a presença de pelo menos uma alteração fonoaudiológica, além da encontrada na fala, apesar da queixa específica, o que evidencia grande preocupação em torno da produção da comunicação oral ${ }^{(24)}$.

Quanto às hipóteses diagnósticas fonoaudiológicas, as mais encontradas foram os desvios fonéticos, seguidos de desvios fonético-fonológicos e de atraso de linguagem. Estes dados coincidem com os de outros estudos; no entanto, com nomenclaturas diferentes. Alguns estudos divulgam maior predominância de diagnósticos de alterações de fala em seus levantamentos ${ }^{(2,23)}$.

As alterações de linguagem foram a segunda hipótese diagnóstica também mais referida em um trabalho ${ }^{(23)}$, diferindo de outro, que encontrou em um dos locais de pesquisa o diagnóstico de alterações de fala associado às alterações de leitura e escrita e em outro local, o de fissura labiopalatina ${ }^{(2)}$. Encontrou-se em outro levantamento que a maior parte da demanda para fonoaudiologia dividiu-se quase que igualmente entre os casos de distúrbio articulatório, distúrbio de leitura e escrita, retardo de linguagem e deficiência auditiva ${ }^{(19)}$. Porém, no presente estudo, não foi encontrada nenhuma hipótese diagnóstica de deficiência auditiva.

Destaque-se a pequena ocorrência de alteração do sistema motor oral $(3,3 \%)$, enquanto que em outros trabalhos foi citada como uma das patologias mais encontradas ${ }^{(26,27)}$. Já a hipótese diagnóstica de gagueira coincide com a citada em outro estudo ${ }^{(22)}$.

O registro nos prontuários de que $15,2 \%$ dos casos encaminhados para o Serviço de Fonoterapia não apresentava indicação para terapia fonoaudiológica, coincide com outro estudo, onde $16 \%$ das crianças encaminhadas apresentavam desenvolvimento da audição e da linguagem esperados para a idade ${ }^{(27)}$.

\section{CONCLUSÃO}

Neste estudo foi possível identificar o perfil da demanda para atendimento fonoaudiológico na área de fonoterapia do Centro de Saúde Santa Marta, Porto Alegre (RS). Destaca-se, entre seus achados, a predominância de encaminhamentos de crianças do gênero masculino e em faixa escolar, similar a outros estudos da área; encaminhados em sua maioria por médico pediatra, com queixa de alteração de fala. A hipótese diagnóstica predominante foi a de desvio fonético, seguida da hipótese de desvio fonético-fonológico, de casos sem indicação para tratamento na especialidade e de prontuários sem hipótese registrada.

Considera-se importante o investimento no planejamento de ações em saúde fonoaudiológica neste município, voltadas, principalmente, à prevenção e à promoção de saúde junto às Unidades Básicas de Saúde e Escolas/Creches pertencentes as suas áreas de abrangência, em programas de educação permanente, articulados ou não aos centros formadores deste profissional. O objetivo deste tipo de ação é que as alterações de fala diminuam a médio e longo prazo, assim como as listas de espera para tal especialidade e que os registros em prontuários e encaminhamentos para esta área ocorram de forma mais qualificada. 


\begin{abstract}
Purpose: To characterize the demand referred to the Speech-Language Pathology and Audiology Service of a Primary Healthcare Center, in the period of 2006/2007. Methods: A transversal report study was carried out using databases and the files of the aforementioned service. From the databases, the following variables were obtained: gender, age and origin of reference. From the patients' files, we obtained data about the initial complaint and the diagnostic hypothesis. Results: From the total number of registries ( $\mathrm{n}=243$ ), the majority regarded male patients (65\%), with ages from six to 12 years $(52.3 \%)$, referred by the pediatrician (35.3\%), with complaints of speech disorders (67.8\%) and diagnostic hypothesis of phonetic deviation (28.4\%); 37 files (15.2\%) had no treatment indication, and $31(12.8 \%)$ did not register a diagnostic hypothesis. Conclusion: More investments on planning speech-language pathology health actions are necessary, in order to obtain medium to long-term decrease of speech disorders, as well as the waiting lists for this specialty, and for the records of the files and referrals to be more appropriate.
\end{abstract}

Keywords: Health sciences; Public health; Health management; Epidemiology; Health services needs and demand

\title{
REFERÊNCIAS
}

1. Andrade CRF de. Fases e níveis de prevenção em fonoaudiologia ações coletivas e individuais. In: Vieira RM, Ávila CRB, Pereira LD, organizadores. Fonoaudiologia e saúde pública. 2a ed. rev. e ampl. Carapicuíba: Pró-Fono; 2000. p.81-104.

2. Gonçalves CGO, Lacerda CBF, Perotino S, Mugnaine AMM. Demanda pelos serviços de fonoaudiologia no município de Piracicaba: estudo comparativo entre a clínica-escola e o atendimento na Prefeitura Municipal. Pró-Fono. 2000;12(2):61-6.

3. Souza RPF, Cunha DA, Silva HJ. Fonoaudiologia: a inserção da área de linguagem no Sistema Único de Saúde (SUS). Rev CEFAC. 2005;7(4):426-32.

4. Mendes VLF. Fonoaudiologia e saúde coletiva: perspectivas de atuação nos serviços públicos de saúde. Distúrb Comun. 1999;10(2):213-24.

5. Lipay MS, Almeida EC. A fonoaudiologia e sua inserção na saúde pública. Rev Ciênc Méd (Campinas). 2007;16(1):31-41.

6. Bernardi APA. Fonoaudiologia na saúde coletiva: uma área em crescimento: editorial. Rev CEFAC. 2007;9(2).

7. Basilio CS, Cardoso RM, Lemos SMA, Pereira LD. Fonoaudiologia e saúde coletiva. Rev Paul Pediatr. 2000;18(4):179-80.

8. Penteado RZ, Servilha EAM. Fonoaudiologia em saúde pública/coletiva: compreendendo prevenção e o paradigma da promoção da saúde. Distúrb Comun. 2004;16(1):107-16.

9. Delgado SE. A fonoaudiologia no contexto da promoção da saúde coletiva materno-infantil. Fono Atual. 2004;7(29):72-5.

10. Befi D. A inserção da fonoaudiologia na atenção primária à saúde. In: Befi $\mathrm{D}$, organizadora. Fonoaudiologia na atenção primária à saúde. São Paulo: Lovise; 1997. p.15-35.

11. Goulart BNG, Chiari BM. Construção e aplicação de indicadores de saúde na perspectiva fonoaudiológica: contribuições para reflexão. Rev Soc Bras Fonoaudiol. 2006;11(3):194-204.

12. Goulart BNG. Contribuição da epidemiologia para a pesquisa e atuação clínica em fonoaudiologia. Fono Atual. 2002;5(21):60-3.

13. Goulart BNG, Chiari BM. Prevalência de desordens de fala em escolares e fatores associados. Rev Saúde Pública. 2007;41(5):726-31

14. Secretaria Municipal de Saúde de Porto Alegre. Levantamento das Listas de Espera para Fonoterapia no Município de Porto Alegre. Porto Alegre: Assessoria de Planejamento e Programação; 2009. Mimeo.

15. Instituto Brasileiro de Geografia e Estatística. Censo Demográfico 2000. [citado 2008 Nov 11]. Disponível em: http://www.ibge.gov.br/home/ estatistica/populacao/default_censo_2000.shtm
16. Tanigute CC. Desenvolvimento das funções estomatognáticas. In: Marchesan IQ. Fundamentos em fonoaudiologia: aspectos clínicos da motricidade oral. Rio de Janeiro: Guanabara Koogan; 1998. p. 1-6.

17. Zorzi JL. Diferenciando alterações da fala e a linguagem. In: Marchesan IQ. Fundamentos em fonoaudiologia: aspectos clínicos da motricidade oral. Rio de Janeiro: Guanabara Koogan; 1998. p. 59-74.

18. Pinho SMR. Avaliação e tratamento da voz. In: Pinho SMR. Fundamentos em fonoaudiologia: tratando os distúrbios da voz. Rio de Janeiro: Guanabara Koogan; 1998. p. 3-37.

19. Freire RM. Fonoaudiologia em saúde pública. Rev Saúde Pública. 1992;26(3):179-84.

20. Silva SA, Fioravanti MP, Martins RHG. Distúrbios articulatórios funcionais. Pró-Fono. 1994;6(1):14-6.

21. Hage SRV, Faiad LNV. Perfil de pacientes com alteração de linguagem atendidos na clínica de diagnóstico dos distúrbios da comunicação - Universidade de São Paulo - Campus Bauru. Rev CEFAC. 2005;7(4):433-40.

22. Lima BPS, Guimarães JATL, Rocha MCG. Características epidemiológicas das alterações de linguagem em um centro fonoaudiológico do primeiro setor. Rev Soc Bras Fonoaudiol. 2008;13(4):376-80.

23. César AM, Maksud SS. Caracterização da demanda de fonoaudiologia no serviço público municipal de Ribeirão das Neves - MG. Rev CEFAC. 2007;9(1):133-8.

24. Franco DP, Ávila CRB. Achados fonoaudiológicos de crianças com queixa de distúrbio de fala. Pró-Fono. 2000;12(1):40-7.

25. Pereira MTJG. Fonoaudiologia: uma vivência em saúde pública [monografia]. São Paulo: CEFAC; 1999.

26. Andrade CRF. Prevalência das desordens idiopáticas da fala e da linguagem em crianças de um a onze anos de idade. Rev Saúde Pública = J Public Health. 1997;31(5):495-501.

27. Ortiz KZ, Bertachini L, Pereira LD. Atuação fonoaudiológica em unidades básicas de saúde. In: Vieira RM, Vieira MM, Ávila CRB, Pereira LD, organizadores. Fonoaudiologia e saúde pública. 2a ed. rev. e ampl. Carapicuíba: Pró-Fono; 2000 p.121-36. 